\title{
Irpexoates A-D, Four Triterpenoids with Malonyl Modifications from the Fruiting Bodies of the Medicinal Fungus Irpex lacteus
}

\author{
Yang Tang ${ }^{1,2,3} \cdot$ Zhen-Zhu Zhao $^{2} \cdot$ Zheng-Hui Li $^{2} \cdot$ Tao Feng $^{2} \cdot$ He-Ping Chen ${ }^{2} \cdot \mathrm{Ji}^{-K a i} \mathrm{Liu}^{2}$
}

Received: 14 February 2018/Accepted: 26 March 2018/Published online: 28 March 2018

(c) The Author(s) 2018

\begin{abstract}
Four eburicane-type triterpenoids with malonyl modifications, namely irpexoates A-D (1-4), were isolated from the fruiting bodies of the medicinal fungus Irpex lacteus. The structures of the new compounds were established by extensive spectroscopic methods, including 1D and 2D NMR, HRESIMS spectroscopic analysis. Irpexoate B (2) displayed weak cytotoxicity against four human cancer cell lines (A-549, SMMC-7721, MCF-7, SW480) with $\mathrm{IC}_{50}$ values varying from 22.9 to $34.0 \mu \mathrm{M}$, and irpexoate D (4) showed weak cytotoxicity against the human cancer cell line SW480 with an $\mathrm{IC}_{50}$ value of $35.2 \mu \mathrm{M}$.
\end{abstract}

Graphical Abstract
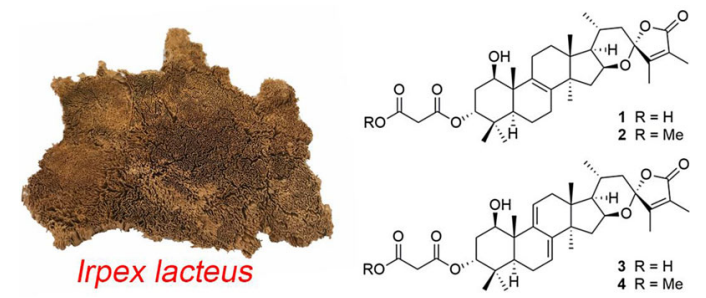

Keywords Irpex lacteus $\cdot$ Meruliaceae $\cdot$ Eburicane $\cdot$ Malonyl $\cdot$ Cytotoxicity

\section{Introduction}

Electronic supplementary material The online version of this article (https://doi.org/10.1007/s13659-018-0160-3) contains supplementary material, which is available to authorized users.

He-Ping Chen

chenhp@mail.scuec.edu.cn

$\bowtie$ Ji-Kai Liu

jkliu@mail.kib.ac.cn

1 State Key Laboratory of Phytochemistry and Plant Resources in West China, Kunming Institute of Botany, Chinese Academy of Sciences, Kunming 650201, People's Republic of China

2 School of Pharmaceutical Sciences, South-Central University for Nationalities, Wuhan 430074, People's Republic of China

3 University of Chinese Academy of Sciences, Beijing 100049, People's Republic of China
Natural products from higher fungi (mushroom) are regarded as a vital source of lead compounds in the field of drug research and development [1]. The fungus Irpex lacteus is widely used in China as a folk medicine [2]. Previously chemical investigation on this fungus major focused on secondary metabolites from culture broth [3, 4], while the study on the fruiting bodies remained untapped.

Triterpenoids are one of the largest groups of secondary metabolites from higher fungi. To the best of our knowledge, lanostane-type triterpenoids are by far the most diverse category of higher fungi-derived triterpenoids. This type of triterpenes always encounters in the genus Ganoderma and the fungus Poria cocos [1]. However, eburicane-type triterpenes, which also can be considered as 24-methyl lanostanes, have rarely been found from fungi. Triterpenoids with malonyl modification are not prevalent 
in fungal metabolites. From biosynthetic point, the malonyl moieties were introduced by the precursor malonyl-CoA, which was a key intermediate in the pathways of fatty acid biosynthesis and fatty acid elongation, and also a key signaling molecule in mammalian cells [5]. As our continuous efforts to search promising lead compounds from mushroom, four malonyl modified eburicanes, namely irpexoates A-D (1-4) (Fig. 1), were isolated from the fruiting bodies of the medicinal fungus I. lacteus. Herein, we report the isolation, structure elucidation, and cytotoxicity against five human cancer lines of the isolates irpexoates A-D (1-4).

\section{Results and Discussion}

Compound 1 was obtained as a white amorphous powder. It had a molecular formula of $\mathrm{C}_{34} \mathrm{H}_{48} \mathrm{O}_{8}$ as determined by (+)-HRESIMS protonated ion peak at $m / z 585.3433$ $[\mathrm{M}+\mathrm{H}]^{+}$(calcd for $\mathrm{C}_{34} \mathrm{H}_{49} \mathrm{O}_{8}, 585.3422$ ), corresponding to $11^{\circ}$ of unsaturation. The IR spectrum showed absorption bands for hydroxy $\left(3436 \mathrm{~cm}^{-1}\right)$ and carbonyl $\left(1764 \mathrm{~cm}^{-1}\right)$ groups. The ${ }^{1} \mathrm{H}$ NMR spectrum of $\mathbf{1}$ (Table 1) showed the presence of seven methyl singlets at $\delta_{\mathrm{H}} 0.86\left(\mathrm{CH}_{3}-18\right), 1.03$ $\left(\mathrm{CH}_{3}-19\right), 1.78\left(\mathrm{CH}_{3}-27\right), 0.87\left(\mathrm{CH}_{3}-28\right), 0.93\left(\mathrm{CH}_{3}-29\right)$, $0.95\left(\mathrm{CH}_{3}-30\right)$, and $1.94\left(\mathrm{CH}_{3}-31\right)$, and one doublet methyl at $\delta_{\mathrm{H}} 1.02\left(\mathrm{~J}=6.0 \mathrm{~Hz}, \mathrm{CH}_{3}-21\right)$. The ${ }^{13} \mathrm{C} \mathrm{NMR}$ and DEPT spectra presented 34 carbons ascribable to eight methyls, eight methylenes, six methines, and twelve quaternary<smiles>[R][R]=C[R]=C</smiles>

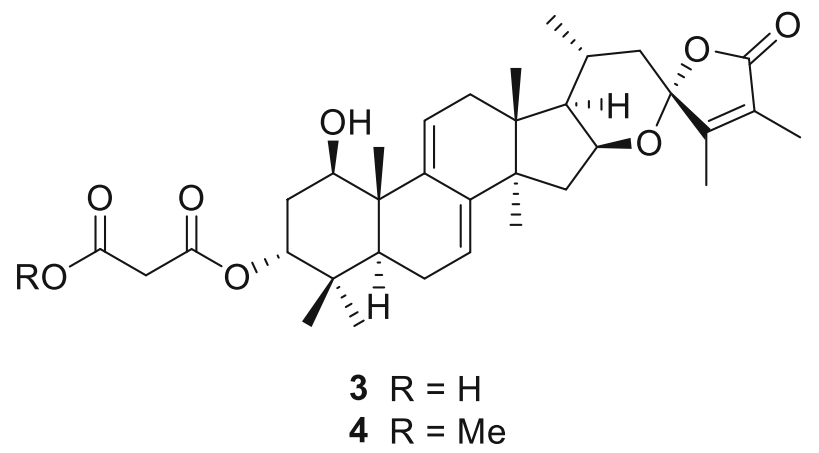

Fig. 1 Chemical structures of compounds 1-4 carbons, including four $\mathrm{sp}^{3}$ ones, four olefinic ones, three carbonyls, and a ketal carbon $\left(\delta_{\mathrm{C}} 107.7, \mathrm{C}-23\right)$. All these spectroscopic features implied the presence of an eburicane skeleton in compound 1. Exhaustive analysis of the 2D NMR spectra furnished the establishment of the structure of 1. The HMBC correlations from $\mathrm{H}_{3}-31$ to $\mathrm{C}-23, \mathrm{C}-24$ $\left(\delta_{\mathrm{C}} 157.8\right)$, and $\mathrm{C}-25\left(\delta_{\mathrm{C}} 124.1\right)$, from $\mathrm{H}_{3}-27$ to $\mathrm{C}-24, \mathrm{C}-25$, and C-26 $\left(\delta_{\mathrm{C}} 172.5\right)$, from $\mathrm{H}-16\left(\delta_{\mathrm{H}} 4.51\right)$ to $\mathrm{C}-23$ (Fig. 2) indicated the existence of a 26,23-lactone, and a 16,23epoxy group, which constructing a spiro ring system with $\mathrm{C}-23$ as the shared carbon. The HMBC correlations from $\mathrm{H}_{3}-19$ to $\mathrm{C}-1\left(\delta_{\mathrm{C}} 71.3\right), \mathrm{C}-9\left(\delta_{\mathrm{C}} 135.1\right)$, from $\mathrm{H}_{3}-28, \mathrm{H}_{3}-29$ to $\mathrm{C}-3\left(\delta_{\mathrm{C}} 81.0\right)$, and from $\mathrm{H}_{3}-30$ to $\mathrm{C}-8$ ( $\left.\delta_{\mathrm{C}} 134.8\right)$ (Fig. 2) revealed that $\mathrm{C}-1$ and $\mathrm{C}-3$ were oxygenated, and a double bond was assigned at the positions C-8-C-9. Apart from the signals assigned to the eburicane skeleton, the remained three carbon signals, i.e. two carbonyls at $\delta_{\mathrm{C}} 167.8\left(\mathrm{C}-1^{\prime}\right)$ and $169.7\left(\mathrm{C}-3^{\prime}\right)$, and a methylene at $\delta_{\mathrm{C}} 41.1\left(\mathrm{C}-2^{\prime}\right)$ were due to a malonyl moiety attached to $\mathrm{C}-3$, which was supported by the HMBC correlations from $\mathrm{H}-2^{\prime}\left(\delta_{\mathrm{H}} 3.46 ; 3.40\right)$ to $\mathrm{C}-1^{\prime}, \mathrm{C}-3^{\prime}$, and from $\mathrm{H}-3\left(\delta_{\mathrm{H}} 4.83\right)$ to $\mathrm{C}-1^{\prime}$.

The ROESY correlations between $\mathrm{H}-1 / \mathrm{H}-5, \mathrm{H}_{3}-30 / \mathrm{H}-16$ demonstrated that $1-\mathrm{OH}$ was $\beta$ orientation while $\mathrm{H}-16$ was $\alpha$ orientation. The key diagnostic ROESY correlation between $\mathrm{H}_{3}-18$ and $\mathrm{H}_{3}-31$ revealed that $\mathrm{C}-23$ was $R^{*}$ configuration (Fig. 3). As for the configuration of C-3, the broad triplet of $\mathrm{H}-3$ with a small coupling constant $(3.0 \mathrm{~Hz})$ indicated the $\beta$ orientation for $\mathrm{H}-3$, which can be seen as a consequence of the nearly same dihedral angle between $\mathrm{H}-3 / \mathrm{H}-2 \alpha$, and $\mathrm{H}-3 / \mathrm{H}-2 \beta$ when $\mathrm{H}-3$ adopted $\beta$ orientation (Fig. 3). Therefore, compound $\mathbf{1}$ was determined as shown in Fig. 1, and given the name irpexoate A.

The white amorphous powder compound $\mathbf{2}$, named irpexoate $\mathrm{B}$, possessed the molecular formula of $\mathrm{C}_{35} \mathrm{H}_{50} \mathrm{O}_{8}$ as determined by the (+)-HRESIMS sodium adduct ion peak at $m / z 621.3399[\mathrm{M}+\mathrm{Na}]^{+}$(calcd for $\mathrm{C}_{35} \mathrm{H}_{50} \mathrm{O}_{8} \mathrm{Na}$, 621.3398), indicating 11 indices of hydrogen deficiency. The ${ }^{1} \mathrm{H}$ and ${ }^{13} \mathrm{C}$ NMR spectra of 2 (Tables 1,2) showed highly similarities to those of $\mathbf{1}$, indicating that these two compounds possessed identical carbon scaffold and substitution patterns. Compared to $\mathbf{1}$, the presence of an additional methoxy group at $\delta_{\mathrm{C}} 52.4\left(\mathrm{C}-4^{\prime}\right)$ in 2 , along with the HMBC correlation from $\mathrm{H}_{3}-4^{\prime}\left(\delta_{\mathrm{H}} 3.72\right)$ to $\mathrm{C}-3^{\prime}\left(\delta_{\mathrm{C}}\right.$ 167.1) (Fig. 2) revealed that the terminal carboxylic acid group was methyl-esterified in $\mathbf{2}$. Other characteristic ROESY correlations suggested that the stereochemistry of chiral centers in 2 was identical with those of $\mathbf{1}$. As describe above, compound $\mathbf{2}$ was determined as shown in Fig. 1.

Compound 3 gave a molecular formula of $\mathrm{C}_{34} \mathrm{H}_{46} \mathrm{O}_{8}$ according to the ${ }^{13} \mathrm{C}$ NMR data and the $(+)$-HRESIMS sodium adduct ion peak at $\mathrm{m} / z, 605.3058[\mathrm{M}+\mathrm{Na}]^{+}$(calcd for $\left.\mathrm{C}_{34} \mathrm{H}_{46} \mathrm{O}_{8} \mathrm{Na}, 605.3085\right)$. A comparison of the ${ }^{1} \mathrm{H}$ and 
Table $1{ }^{1} \mathrm{H}$ NMR spectroscopic data for compounds $\mathbf{1 - 4}\left(\mathrm{CDCl}_{3}\right)$

\begin{tabular}{|c|c|c|c|c|}
\hline No. & $\mathbf{1}^{\mathrm{a}}$ & $2^{\mathrm{a}}$ & $\mathbf{3}^{\mathrm{a}}$ & $4^{b}$ \\
\hline 1 & $3.82, \mathrm{dd}(9.0,6.4)$ & $3.80, \mathrm{dd}(10.0,6.3)$ & $4.10, \mathrm{dd}(10.0,6.3)$ & 4.09, dd $(10.0,6.3)$ \\
\hline 2 & 1.92, overlapped & $1.92, \mathrm{~m}$ & 1.98, overlapped & 1.96, overlapped \\
\hline 3 & 4.83 , br t (3.0) & $4.81, \mathrm{t}(3.0)$ & 4.85 , br t (3.0) & $4.80, \mathrm{t}(3.0)$ \\
\hline 5 & $1.46, \mathrm{dd}(10.0,6.0)$ & $1.44, \mathrm{dd}(10.0,6.0)$ & 1.51, dd $(12.0,4.0)$ & $1.49, \mathrm{dd}(12.0,4.0)$ \\
\hline \multirow[t]{2}{*}{6} & 1.63, overlapped & 1.64, overlapped & 2.15 , overlapped & $2.14, \mathrm{dd}(18.8,14.0)$ \\
\hline & & & 2.06, overlapped & 2.06, overlapped \\
\hline \multirow[t]{2}{*}{7} & $2.09, \mathrm{~m}$ & 2.10, overlapped & $5.49, \mathrm{~d}(6.5)$ & $5.49, \mathrm{~d}(6.3)$ \\
\hline & $2.04, \mathrm{~m}$ & 2.04, overlapped & & \\
\hline \multirow[t]{2}{*}{11} & $2.43, \mathrm{~m}$ & 2.44 , overlapped & $6.30, \mathrm{~d}(6.0)$ & $6.30, \mathrm{~d}(6.0)$ \\
\hline & 2.21, br dd $(19.0,8.5)$ & 2.23 , overlapped & & \\
\hline \multirow[t]{2}{*}{12} & 1.84, overlapped & 1.85, overlapped & $2.24, \mathrm{~d}(17.5)$ & $2.24, \mathrm{~d}(17.7)$ \\
\hline & 1.62 , overlapped & 1.64, overlapped & 2.08, overlapped & 2.09 , overlapped \\
\hline \multirow[t]{2}{*}{15} & $1.88, \mathrm{dd}(12.4,8.0)$ & $1.88, \mathrm{dd}(12.5,7.8)$ & 2.06, overlapped & 2.06, overlapped \\
\hline & $1.70, \mathrm{dd}(12.4,6.5)$ & $1.71, \mathrm{dd}(12.5,6.5)$ & $1.71, \mathrm{dd}(12.5,6.5)$ & $1.71, \mathrm{dd}(12.5,6.5)$ \\
\hline 16 & 4.51, ddd $(8.0,8.0,6.5)$ & 4.51, ddd $(7.8,7.8,6.5)$ & 4.59 , ddd $(7.8,7.8,6.5)$ & 4.59, ddd $(7.8,7.8,6.5)$ \\
\hline 17 & 1.59 , overlapped & 1.59 , overlapped & 1.67, overlapped & $1.66, \mathrm{~m}$ \\
\hline 18 & $0.86, \mathrm{~s}$ & $0.86, \mathrm{~s}$ & $0.75, \mathrm{~s}$ & $0.75, \mathrm{~s}$ \\
\hline 19 & $1.03, \mathrm{~s}$ & $1.02, \mathrm{~s}$ & $1.07, \mathrm{~s}$ & $1.07, \mathrm{~s}$ \\
\hline 20 & 1.81, overlapped & 1.81, overlapped & 1.82, overlapped & 1.81, overlapped \\
\hline 21 & $1.02, \mathrm{~d}(6.0)$ & $1.03, \mathrm{~d}(6.0)$ & $1.03, \mathrm{~d}(6.5)$ & $1.02, \mathrm{~d}(6.5)$ \\
\hline 22 & 1.81, overlapped & 1.81, overlapped & 1.82, overlapped & 1.82, overlapped \\
\hline 27 & $1.78, \mathrm{~s}$ & $1.78, \mathrm{~s}$ & $1.78, \mathrm{~s}$ & $1.78, \mathrm{~s}$ \\
\hline 28 & $0.87, \mathrm{~s}$ & $0.87, \mathrm{~s}$ & $0.89, \mathrm{~s}$ & $0.88, \mathrm{~s}$ \\
\hline 29 & $0.93, \mathrm{~s}$ & $0.93, \mathrm{~s}$ & $1.01, \mathrm{~s}$ & $1.00, \mathrm{~s}$ \\
\hline 30 & $0.95, \mathrm{~s}$ & $0.94, \mathrm{~s}$ & $0.93, \mathrm{~s}$ & $0.92, \mathrm{~s}$ \\
\hline 31 & $1.94, \mathrm{~s}$ & $1.94, \mathrm{~s}$ & $1.93, \mathrm{~s}$ & $1.93, \mathrm{~s}$ \\
\hline \multirow[t]{2}{*}{$2^{\prime}$} & $3.46, \mathrm{~d}(16.5)$ & $3.43, \mathrm{~d}(16.9)$ & $3.46, \mathrm{~d}(17.0)$ & $3.46, \mathrm{~d}(17.0)$ \\
\hline & $3.40, \mathrm{~d}(16.5)$ & $3.39, \mathrm{~d}(16.9)$ & $3.41, \mathrm{~d}(17.0)$ & $3.41, \mathrm{~d}(17.0)$ \\
\hline $4^{\prime}$ & & $3.73, \mathrm{~s}$ & & $3.70, \mathrm{~s}$ \\
\hline
\end{tabular}

${ }^{\mathrm{a}}$ Measured at $500 \mathrm{MHz}$

${ }^{\mathrm{b}}$ Measured at $600 \mathrm{MHz}$

${ }^{13} \mathrm{C}$ NMR data of $\mathbf{3}$ to those of $\mathbf{1}$ (Tables 1,2 ) revealed that $\mathbf{3}$ was an analogue of $\mathbf{1}$. Carefully analysis of the HMBC and ${ }^{1} \mathrm{H}-{ }^{1} \mathrm{H}$ COSY spectra of 3 revealed the existence of a conjugated diene locating at C-7-C-8-C-9-C-11, which was ascertainable by the HMBC correlations from the olefinic protons $\mathrm{H}-7\left(\delta_{\mathrm{H}} 5.49\right)$ and $\mathrm{H}-11\left(\delta_{\mathrm{H}} 6.30\right)$ to $\mathrm{C}-8$ $\left(\delta_{\mathrm{C}} 141.8\right), \mathrm{C}-9\left(\delta_{\mathrm{C}} 144.2\right)$ as well as the ${ }^{1} \mathrm{H}-{ }^{1} \mathrm{H}$ COSY cross peaks between $\mathrm{H}-6 / \mathrm{H}-7, \mathrm{H}-11 / \mathrm{H}-12$ (Fig. 2). The stereochemistry of $\mathbf{3}$ was assigned by a ROESY experiment and coupling constants analysis. The diagnostic ROESY correlations between $\mathrm{H}-1 / \mathrm{H}-5, \mathrm{H}_{3}-18 / \mathrm{H}-20, \mathrm{H}_{3}-18 / \mathrm{H}_{3}-31$, $\mathrm{H}_{3}-30 / \mathrm{H}-16 / \mathrm{H}-17$ indicated that both $\mathrm{H}-1$ and $\mathrm{H}-16$ were $\alpha$ orientation, and the spiro carbon $\mathrm{C}-23$ was $R^{*}$ configuration (Fig. 3). The triplet peak of $\mathrm{H}-3\left(\delta_{\mathrm{H}} 4.85\right)$ with small coupling constant $(3.0 \mathrm{~Hz})$ suggested the malonate moiety at the axial position. Therefore, compound $\mathbf{3}$ was established as shown in Fig. 1 and given the trivial name irpexoate $\mathrm{C}$.

A molecular formula of $\mathrm{C}_{35} \mathrm{H}_{48} \mathrm{O}_{8}$ was assigned to compound $\mathbf{4}$ as determined by the (+)-HRESIMS sodium adduct ion peak at $\mathrm{m} / \mathrm{z} 619.3250[\mathrm{M}+\mathrm{Na}]^{+}$(calcd for $\left.\mathrm{C}_{35} \mathrm{H}_{48} \mathrm{O}_{8} \mathrm{Na}, 619.3241\right)$. Comparison of the 1D NMR data of $\mathbf{4}$ (Tables 1,2) to those of $\mathbf{3}$ showed that they were closely related analogues featuring identical carbon frameworks. 2D NMR spectra analysis of $\mathbf{4}$ indicated that the main distinction was attributable to the substituent group at C-3. The HMBC correlations from $\mathrm{H}-3\left(\delta_{\mathrm{H}} 4.80\right)$ to $\mathrm{C}-1^{\prime}\left(\delta_{\mathrm{C}} 165.9\right)$, from $\mathrm{H}-2^{\prime}\left(\delta_{\mathrm{H}} 3.41,3.46\right)$ to $\mathrm{C}-1^{\prime}$ and $\mathrm{C}-3^{\prime}\left(\delta_{\mathrm{C}} 166.8\right)$, and $\mathrm{CH}_{3}-4^{\prime}\left(\delta_{\mathrm{H}} 3.70\right)$ to $\mathrm{C}-3^{\prime}$ allowed a methyl malonate moiety linked at $\mathrm{C}-3$. The relative 

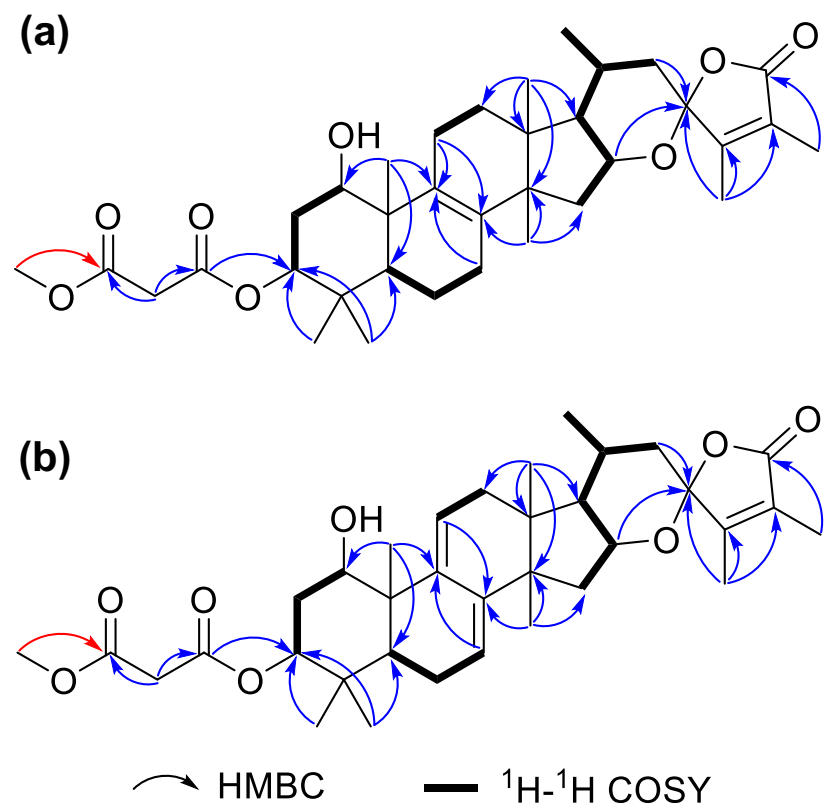

Fig. 2 Characteristic mutual HMBC (blue arrow) and ${ }^{1} \mathrm{H}-{ }^{1} \mathrm{H}$ COSY correlations for a compounds $\mathbf{1}$ and $\mathbf{2}$ (HMBC in red arrow was exclusive for $\mathbf{2}$ ) and $\mathbf{b}$ compounds $\mathbf{3}$ and $\mathbf{4}$ (HMBC in red arrow was exclusive for $\mathbf{4}$ )
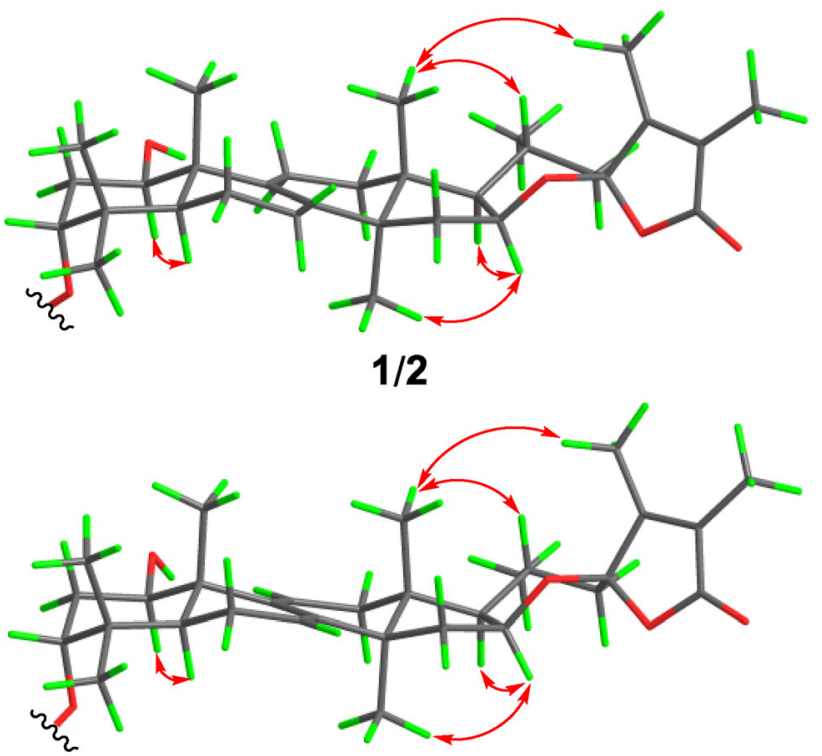

$3 / 4$

\section{$\sim$ ROESY}

Fig. 3 Key ROESY correlations for compounds 1-4

configuration of $\mathbf{4}$ was consistent with that of $\mathbf{3}$ as established by the ROESY spectrum. The structure of $\mathbf{4}$ was consequently assigned as shown in Fig. 1, and named as irpexoate D.

Irpexoates A-D (1-4) were evaluated for the cytotoxicity against five human cancer cell lines (the human
Table $2{ }^{13} \mathrm{C}$ NMR spectroscopic data for compounds $\mathbf{1}-\mathbf{4}\left(\mathrm{CDCl}_{3}\right)$

\begin{tabular}{|c|c|c|c|c|}
\hline No. & $\mathbf{1}^{\mathrm{a}}$ & $2^{\mathrm{a}}$ & $3^{\mathrm{a}}$ & $4^{\mathrm{b}}$ \\
\hline 1 & $71.3, \mathrm{CH}$ & $71.3, \mathrm{CH}$ & 71.6, CH & 71.6, CH \\
\hline 2 & $33.4, \mathrm{CH}_{2}$ & $33.5, \mathrm{CH}_{2}$ & $33.5, \mathrm{CH}_{2}$ & $33.7, \mathrm{CH}_{2}$ \\
\hline 3 & $81.0, \mathrm{CH}$ & $80.4, \mathrm{CH}$ & $81.0, \mathrm{CH}$ & $80.3, \mathrm{CH}$ \\
\hline 4 & $36.5, \mathrm{C}$ & $36.6, \mathrm{C}$ & $36.4, \mathrm{C}$ & $36.5, \mathrm{C}$ \\
\hline 5 & $44.4, \mathrm{CH}$ & $44.3, \mathrm{CH}$ & $43.9, \mathrm{CH}$ & $43.9, \mathrm{CH}$ \\
\hline 6 & $17.9, \mathrm{CH}_{2}$ & $17.8, \mathrm{CH}_{2}$ & $23.1, \mathrm{CH}_{2}$ & $23.1, \mathrm{CH}_{2}$ \\
\hline 7 & 26.0, $\mathrm{CH}_{2}$ & 26.1, $\mathrm{CH}_{2}$ & $120.2, \mathrm{CH}$ & $120.4, \mathrm{CH}$ \\
\hline 8 & 134.8, C & 134.8, C & $141.8, \mathrm{C}$ & $141.8, \mathrm{C}$ \\
\hline 9 & 135.1, C & 135.2, C & $144.2, \mathrm{C}$ & $144.3, \mathrm{C}$ \\
\hline 10 & 43.0, C & 43.1, C & $43.4, \mathrm{C}$ & 43.5, C \\
\hline 11 & 23.9, $\mathrm{CH}_{2}$ & 23.9, $\mathrm{CH}_{2}$ & $119.8, \mathrm{CH}$ & $119.8, \mathrm{CH}$ \\
\hline 12 & 31.6, $\mathrm{CH}_{2}$ & 31.6, $\mathrm{CH}_{2}$ & 37.6, $\mathrm{CH}_{2}$ & 37.6, $\mathrm{CH}_{2}$ \\
\hline 13 & $43.4, \mathrm{C}$ & $43.4, \mathrm{C}$ & $42.8, \mathrm{C}$ & $42.8, \mathrm{C}$ \\
\hline 14 & $47.4, \mathrm{C}$ & 47.4, C & 47.9, C & $48.0, \mathrm{C}$ \\
\hline 15 & $39.5, \mathrm{CH}_{2}$ & $39.5, \mathrm{CH}_{2}$ & $40.0, \mathrm{CH}_{2}$ & $40.1, \mathrm{CH}_{2}$ \\
\hline 16 & $75.5, \mathrm{CH}$ & $75.4, \mathrm{CH}$ & $75.2, \mathrm{CH}$ & $75.2, \mathrm{CH}$ \\
\hline 17 & $53.0, \mathrm{CH}$ & $53.0, \mathrm{CH}$ & $53.5, \mathrm{CH}$ & $53.5, \mathrm{CH}$ \\
\hline 18 & $18.4, \mathrm{CH}_{3}$ & $18.3, \mathrm{CH}_{3}$ & $18.4, \mathrm{CH}_{3}$ & $18.4, \mathrm{CH}_{3}$ \\
\hline 19 & $13.8, \mathrm{CH}_{3}$ & $13.7, \mathrm{CH}_{3}$ & $15.2, \mathrm{CH}_{3}$ & $15.2, \mathrm{CH}_{3}$ \\
\hline 20 & $26.3, \mathrm{CH}$ & $26.3, \mathrm{CH}$ & $26.1, \mathrm{CH}$ & $26.1, \mathrm{CH}$ \\
\hline 21 & $20.8, \mathrm{CH}_{3}$ & $20.8, \mathrm{CH}_{3}$ & 20.6, $\mathrm{CH}_{3}$ & 20.6, $\mathrm{CH}_{3}$ \\
\hline 22 & $37.5, \mathrm{CH}_{2}$ & $37.5, \mathrm{CH}_{2}$ & $37.4, \mathrm{CH}_{2}$ & $37.5, \mathrm{CH}_{2}$ \\
\hline 23 & 107.7, C & 107.6, C & 107.6, C & 107.5, C \\
\hline 24 & 157.8, C & 157.8, C & 157.7, C & 157.7, C \\
\hline 25 & 124.1, C & 124.1, C & 124.1, C & 124.1, C \\
\hline 26 & $172.5, \mathrm{C}$ & $172.5, \mathrm{C}$ & $172.5, \mathrm{C}$ & $172.3, \mathrm{C}$ \\
\hline 27 & $8.3, \mathrm{CH}_{3}$ & $8.3, \mathrm{CH}_{3}$ & $8.3, \mathrm{CH}_{3}$ & $8.3, \mathrm{CH}_{3}$ \\
\hline 28 & $27.2, \mathrm{CH}_{3}$ & $27.2, \mathrm{CH}_{3}$ & 27.5, $\mathrm{CH}_{3}$ & $27.4, \mathrm{CH}_{3}$ \\
\hline 29 & $21.5, \mathrm{CH}_{3}$ & 21.5, $\mathrm{CH}_{3}$ & $22.2, \mathrm{CH}_{3}$ & $22.2, \mathrm{CH}_{3}$ \\
\hline 30 & $25.2, \mathrm{CH}_{3}$ & $25.2, \mathrm{CH}_{3}$ & $25.9, \mathrm{CH}_{3}$ & $25.8 . \mathrm{CH}_{3}$ \\
\hline 31 & $10.9, \mathrm{CH}_{3}$ & $10.9, \mathrm{CH}_{3}$ & $10.8, \mathrm{CH}_{3}$ & $10.9, \mathrm{CH}_{3}$ \\
\hline $1^{\prime}$ & $167.8, \mathrm{C}$ & $165.9, \mathrm{C}$ & 167.7, C & $165.9, \mathrm{C}$ \\
\hline $2^{\prime}$ & $41.1, \mathrm{CH}_{2}$ & $41.7, \mathrm{CH}_{2}$ & $40.4, \mathrm{CH}_{2}$ & 41.6, $\mathrm{CH}_{2}$ \\
\hline $3^{\prime}$ & 169.7, C & 167.1, C & 168.1, C & $166.8, \mathrm{C}$ \\
\hline $4^{\prime}$ & & $52.4, \mathrm{CH}_{3}$ & & $52.4, \mathrm{CH}_{3}$ \\
\hline
\end{tabular}

${ }^{\mathrm{a}}$ Measured at $125 \mathrm{MHz}$

${ }^{\mathrm{b}}$ Measured at $150 \mathrm{MHz}$

myeloid leukemia cell line HL-60, the human hepatocellular carcinoma cell line SMMC-7721, the lung cancer cell line A549, the breast cancer cell line MCF-7, and the human colon cancer cell line SW-480). The results showed that compound $\mathbf{2}$ exhibited weak cytotoxicity against the four human cancer cell lines (A-549, SMMC-7721, MCF7 , SW480) with $\mathrm{IC}_{50}$ values varying from 22.9 to $34.0 \mu \mathrm{M}$, while compound $\mathbf{4}$ only showed weak cytotoxicity against the human colon cancer cell line SW480 with an $\mathrm{IC}_{50}$ value 
of $11.2 \pm 0.25 \mu \mathrm{M}$ (Table 3). However, compounds $\mathbf{1}$ and 3 were devoid of cytotoxicity. Thus, the conclusion could be drawn that the absence of methoxy group in the malonyl moiety attenuated the cytotoxicity.

\section{Experimental}

\subsection{General Experimental Procedures}

Optical rotations were obtained on a JASCO P-1020 digital polarimeter (Horiba, Kyoto, Japan). UV spectra were recorded on a Shimadzu UV-2401PC UV-visible recording spectrophotometer (Shimadzu, Kyoto, Japan). A Tenor 27 spectrophotometer (Bruker Optics GmbH, Ettlingen, Germany) was used for scanning IR spectroscopy using $\mathrm{KBr}$ pellets. 1D and 2D NMR spectra were obtained on Bruker DRX $500 \mathrm{MHz}$ and Avance III $600 \mathrm{MHz}$ spectrometers (Bruker Corporation, Karlsruhe, Germany). HRESIMS were recorded on an Agilent 6200 Q-TOF MS system (Agilent Technologies, Santa Clara, CA, USA). Sephadex LH-20 (Amersham Biosciences, Uppsala, Sweden) and silica gel (Qingdao Haiyang Chemical Co., Ltd, Qingdao, China) were used for column chromatography (CC). Medium pressure liquid chromatography (MPLC) was performed on a Büchi Sepacore System equipped with pump manager C-615, pump modules C-605 and fraction collector C-660 (Büchi Labortechnik AG, Flawil, Switzerland), and columns packed with Chromatorex C-18 (dimensions $450 \mathrm{~mm} \times$ i.d. $14 \mathrm{~mm}$, particle size: 40-75 $\mu \mathrm{m}$, Fuji Silysia Chemical Ltd., Kasugai, Japan). Preparative high performance liquid chromatography (prep-HPLC) were performed on an Agilent 1260 liquid chromatography system equipped with a Zorbax SB-C18 column (particle size $5 \mu \mathrm{m}$, dimension $150 \mathrm{~mm} \times$ i.d.

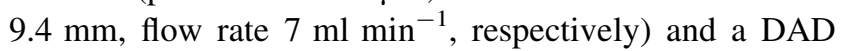
detector (Agilent Technologies).

\subsection{Fungal Material}

The fungus Irpex lacteus was collected from the Wangtianshu Scenic Area, Xishuangbanna, Yunnan Province in July 2014, and authenticated by Prof. Yu-Cheng Dai (Beijing Forestry University), who is a mushroom specialist. A voucher specimen of I. lacteus was deposited at the Mushroom Bioactive Natural Products Research Group in Kunming Institute of Botany (No. HFG 201407).

\subsection{Extraction and Isolation}

The dry fruiting bodies of I. lacteus (1.47 kg) was pulverized and macerated five times with $95 \% \mathrm{EtOH}$ at room temperature. The extract was evaporated under reduced pressure and partitioned between EtOAc and water for three times to give an EtOAc layer (53 g). The crude extract was eluted on MPLC with a stepwise gradient of $\mathrm{MeOH} / \mathrm{H}_{2} \mathrm{O}(20-100 \%)$ to afford eight fractions (A-H).

Fraction E was applied to silica gel column chromatography eluting with petroleum ether/acetone (5:1-2:1) to give ten subfractions (E1-E10). Subfraction E5 was purified by prep-HPLC $\left(\mathrm{MeCN} / \mathrm{H}_{2} \mathrm{O}: 45-65 \%, 7 \mathrm{~mL}\right.$ $\left.\min ^{-1}, 20 \mathrm{~min}\right)$ to yield compound $4\left(1.8 \mathrm{mg}, \mathrm{t}_{R}\right.$ $=13.8 \mathrm{~min})$. Fraction $\mathrm{F}$ was subjected to normal silica gel $\mathrm{CC}\left(\mathrm{CHCl}_{3} / \mathrm{MeOH}, 40: 1-1: 1\right)$ to furnish seven subfractions (F1-F7). Subfraction F6 was purified on prep-HPLC (MeCN/H $\mathrm{H}_{2} \mathrm{O}: 65 \%$, isocratic, $18 \mathrm{~mL} \mathrm{~min}^{-1}, 20 \mathrm{~min}$ ) to yield compound 1 (26.6 $\left.\mathrm{mg}, \mathrm{t}_{R}=9.5 \mathrm{~min}\right)$. Compound 2 (17.0 $\left.\mathrm{mg}, \mathrm{t}_{R}=12.9 \mathrm{~min}\right)$ was purified from subfraction $\mathrm{F} 1$ by prep-HPLC $\left(\mathrm{MeCN} / \mathrm{H}_{2} \mathrm{O}: 55-75 \%, 7 \mathrm{~mL} \mathrm{~min}^{-1}\right.$, 20 min). Subfraction F4 was fractionated into five subfractions (F4a-F4e) by Sephadex LH-20, eluting with $\mathrm{CHCl}_{3}-\mathrm{MeOH}$ (1:1). Subfraction F4e was separated using silica gel $\mathrm{CC}\left(\mathrm{CHCl}_{3} / \mathrm{MeOH}, 100: 1-10: 1\right)$ to afford eight subfractions (F4e1-F4e8). Subfraction F4e5 was fractionated by Sephadex LH-20 (acetone) to give six subfractions (F4e5a-F4e5f). Subfraction F4e5c was further purified using prep-HPLC with a solvent gradient of $45-65 \%$ $\mathrm{MeCN}$ in $\mathrm{H}_{2} \mathrm{O}$ over $20 \mathrm{~min}$, flow rate $7 \mathrm{ml} \cdot \mathrm{min}^{-1}$, to yield compound 3 (3.0 $\left.\mathrm{mg}, \mathrm{t}_{R}=12.9 \mathrm{~min}\right)$.

\subsection{Spectroscopic Data of Compounds}

\subsubsection{Irpexoate A (1)}

Amorphous white powder; $[\alpha]_{\mathrm{D}}^{24}-24.6$ (c $\left.0.15, \mathrm{MeOH}\right)$; $\mathrm{UV}(\mathrm{MeOH}) \lambda_{\max }(\log \varepsilon) 206.4$ (4.23); IR (KBr) $v_{\max } 3436$, 2960, 2933, 2878, 1764, 1612, 1381, 1314, 1158, 1036, $958 \mathrm{~cm}^{-1} ;{ }^{1} \mathrm{H} \mathrm{NMR}\left(500 \mathrm{MHz}, \mathrm{CDCl}_{3}\right)$ and ${ }^{13} \mathrm{C} \mathrm{NMR}$ (125 MHz, $\mathrm{CDCl}_{3}$ ) data, see Tables 1 and 2; HRESIMS $\mathrm{m} /$ z $585.3433[\mathrm{M}+\mathrm{H}]^{+}$(calcd for $\mathrm{C}_{34} \mathrm{H}_{49} \mathrm{O}_{8}, 585.3422$ ).
Table 3 Cytotoxicity of compounds $\mathbf{2}$ and $\mathbf{4}$ against five human cancer cell lines $\left(\mathrm{IC}_{50} \pm \mathrm{SD}\right.$ in $\left.\mu \mathrm{M}\right)$

\begin{tabular}{llllll}
\hline Compound & HL-60 & A-549 & SMMC-7721 & MCF-7 & SW480 \\
\hline $\mathbf{2}$ & $>40$ & $22.9 \pm 0.92$ & $30.1 \pm 0.43$ & $24.2 \pm 1.11$ & $34.0 \pm 1.43$ \\
$\mathbf{4}$ & $>40$ & $>40$ & $>40$ & $>40$ & $35.2 \pm 1.15$ \\
Cisplatin & & $14.1 \pm 0.11$ & $11.9 \pm 0.49$ & $14.1 \pm 1.00$ & $11.2 \pm 0.25$ \\
\hline
\end{tabular}




\subsubsection{Irpexoate B (2)}

Amorphous white powder; $[\alpha]_{\mathrm{D}}^{24}-23.5($ c $0.15, \mathrm{MeOH})$; IR (KBr) $v_{\max } 3439,2959,2878,1753,1631,1439,1313$, 1033, $957 \mathrm{~cm}^{-1}$; ${ }^{1} \mathrm{H}$ NMR $\left(500 \mathrm{MHz}, \mathrm{CDCl}_{3}\right)$ and ${ }^{13} \mathrm{C}$ NMR $\left(125 \mathrm{MHz}, \mathrm{CDCl}_{3}\right)$ data, see Tables 1 and 2; HRESIMS $\mathrm{m} / \mathrm{z} 621.3399[\mathrm{M}+\mathrm{Na}]^{+}$(calcd for $\mathrm{C}_{35} \mathrm{H}_{50} \mathrm{O}_{8} \mathrm{Na}$, 621.3398)

\subsubsection{Irpexoate C (3)}

Amorphous white powder; $[\alpha]_{\mathrm{D}}^{24}-4.0(c 0.15, \mathrm{MeOH}) ; \mathrm{UV}$ $(\mathrm{MeOH}) \lambda_{\max }(\log \varepsilon) 209.0$ (4.17), 232.6 (4.05), 242.0 (4.06) and 250.0 (3.93); IR (KBr) $v_{\max } 3438,2963,2932$, 2883, 1765, 1633, 1315, 1156, 1038, $958 \mathrm{~cm}^{-1} ;{ }^{1} \mathrm{H}$ NMR (500 $\left.\mathrm{MHz}, \mathrm{CDCl}_{3}\right)$ and ${ }^{13} \mathrm{C} \mathrm{NMR}\left(125 \mathrm{MHz}, \mathrm{CDCl}_{3}\right)$ data, see Tables 1 and 2; HRESIMS $\mathrm{m} / \mathrm{z}, 605.3058[\mathrm{M}+\mathrm{Na}]^{+}$ (calcd for $\mathrm{C}_{34} \mathrm{H}_{46} \mathrm{O}_{8} \mathrm{Na}, 605.3085$ ).

\subsubsection{Irpexoate D (4)}

Amorphous white powder; $[\alpha]_{\mathrm{D}}^{24}-4.8(c 0.09, \mathrm{MeOH})$; IR $(\mathrm{KBr}) v_{\max } 3444,2961,2928,2884,1752,1636,1444$, 1314, 1153, 1036, $958 \mathrm{~cm}^{-1}$; ${ }^{1} \mathrm{H}$ NMR $\left(600 \mathrm{MHz}, \mathrm{CDCl}_{3}\right)$ and ${ }^{13} \mathrm{C}$ NMR (150 MHz, $\mathrm{CDCl}_{3}$ ) data, see Tables 1 and 2; HRESIMS $\mathrm{m} / z 619.3250[\mathrm{M}+\mathrm{Na}]^{+}$(calcd for $\mathrm{C}_{35} \mathrm{H}_{48} \mathrm{O}_{8-}$ $\mathrm{Na}, 619.3241)$.

\subsection{Cytotoxicity Assays}

Human myeloid leukemia HL-60 cells, lung cancer A-549 cells, hepatocellular carcinoma SMMC-7721 cells, breast cancer MCF-7 cells, and colon cancer SW480 cell lines were used in the cytotoxic assay. All the cells were cultured in DMEM or RPMI-1640 medium (Hyclone, USA), supplemented with $10 \%$ fetal bovine serum (Hyclone, USA) in $5 \% \mathrm{CO}_{2}$ at $37{ }^{\circ} \mathrm{C}$. The cytotoxicity assay was performed according to the MTS (3-(4,5-dimethylthiazol2-yl)-5(3-carboxymethoxyphenyl)-2-(4-sulfopheny)-2 $\mathrm{H}$-tetrazolium) method in 96-well microplates. Briefly, $100 \mu \mathrm{L}$ of adherent cells was seeded into each well of 96-well cell culture plates and allowed to adhere for $12 \mathrm{~h}$ before drug addition, while suspended cells were seeded just before drug addition to an initial density of $1 \times 105$ cells ml $^{-1}$. Each tumor cell line was exposed to the tested compounds dissolved in DMSO at various concentrations in triplicate for $48 \mathrm{~h}$, with cisplatin (Sigma, USA) as a positive control. After compound treatment, cell viability was detected and a cell growth curve was graphed. $\mathrm{IC}_{50}$ values were calculated by Reed and Muench's method [6].

Acknowledgements This work was financially supported by National Natural Science Foundation of China (81561148013), the Key Projects of Technological Innovation of Hubei Province (2016ACA138), and the Fundamental Research Funds for the Central University, South-Central University for Nationalities (CZT18014, CZT18013). We thank Analytical and Measuring Center, School of Pharmaceutical Sciences, South-Central Univerisity for Nationalities for MS and NMR spectra tests.

\section{Compliance with Ethical Standards}

Conflict of interest The authors declare no conflict of interest.

Open Access This article is distributed under the terms of the Creative Commons Attribution 4.0 International License (http://creative commons.org/licenses/by/4.0/), which permits unrestricted use, distribution, and reproduction in any medium, provided you give appropriate credit to the original author(s) and the source, provide a link to the Creative Commons license, and indicate if changes were made.

\section{References}

1. H.P. Chen, J.K. Liu, Prog. Chem. Org. Nat. Prod. 106, 1-201 (2017)

2. X.M. Dong, X.H. Song, K.B. Liu, C.H. Dong, Mycosystema 36, 28-34 (2017)

3. J.H. Ding, Z.H. Li, T. Feng, J.K. Liu, Fitoterapia 125, 245-248 (2018)

4. J.H. Ding, T. Feng, B.K. Cui, K. Wei, Z.H. Li, J.K. Liu, Tetrahedron Lett. 54, 2651-2654 (2013)

5. D. Saggerson, Annu. Rev. Nutr. 28, 253-272 (2008)

6. H.P. Chen, Z.Z. Zhao, Z.H. Li, Z.J. Dong, K. Wei, X. Bai, L. Zhang, C.N. Wen, T. Feng, J.K. Liu, Chem. Open 5, 142-149 (2016) 